Journal of Universal Computer Science, vol. 20, no. 13 (2014), 1708-1711

submitted: 15/11/14, accepted: 24/11/14, appeared: 28/11/14 @ J.UCS

\title{
Collaborative Computing and Applications
}

\section{J.UCS Special Issue}

\author{
Weiming Shen \\ (Tongji University, Shanghai, P.R. China \\ wshen@ieee.org) \\ Weidong Li \\ (Coventry University, Coventry, United Kingdom \\ aa3719@coventry.ac.uk) \\ Jose Alberto Pino \\ (University of Chile, DCC, Santiago, Chile \\ pinoemh@gmail.com) \\ Junzhou Luo \\ (Southeast University, Nanjing, P.R. China \\ jluo@seu.edu.cn)
}

\section{Introduction}

Evolving from parallel computing, cluster computing, grid computing, to nowadays cloud computing, the distributed computing paradigm has been shifting from parallelization, distribution, sharing, to be able to support full-scale collaboration between systems and people. This new computing paradigm can be called collaborative computing, composing of technologies and techniques to facilitate people, software, and hardware working together via computer-assisted means. A number of well-known technologies are contributing to the emergence of this new computing paradigm, including computer supported cooperative work (CSCW) and groupware, software agents and multi-agent systems, web services and semantic web, social networks and ad-hoc networks. This collaborative computing paradigm and related technologies have seen wide applications in many areas. At the same time, it also offers a lot of research and development opportunities across a number of disciplines and has attracted a lot of attention of many researchers around the world. This Special Issue includes original research papers that report recent advances in collaborative computing technologies and applications.

\section{Contributions of the Special Issue}

We invited the authors of 11 high quality papers presented at the 2013 17th IEEE International Conference on Computer Supported Cooperative Work in Design (CSCWD 2013), to submit extended versions of their contributions to this Special Issue. In addition, an open call for submissions was issued. A total of 16 submissions was received for this Special Issue. Each submission was reviewed by three 
international experts, and a second round of reviews was followed to ensure that the papers were thoroughly improved with the reviewer comments. Finally seven papers were accepted for publication in this Special Issue. These papers cover a wide range of topics in collaborative computing research and development, including synchronization, volunteer computing, collaborative planning information delivery, service discovery and integration, ontology based emotion description, opportunistic networks, and wireless mesh networks.

Synchronization is essential in all collaborative computing systems. Baloian et al. proposed an architecture to support the design and development of distributed collaborative applications using HTML5. A middleware called Coupled Objects was used to support the synchronization by dynamically coupling arbitrary user interface objects between heterogeneous applications. The proposed approach was demonstrated through the design and implementation of four different collaborative systems.

Volunteer computing is a paradigm in which devices participating in a distributed environment share part of their resources to help others to better perform their activities. The effectiveness of this computing paradigm depends on the collaboration attitude adopted by the participating devices. Vega et al. presented a study that helps designers understand the impact of adopting a particular collaboration attitude to contribute with local resources to the distributed shared environment. The study considers five collaboration strategies which are analyzed in computing environments with both abundance and scarcity of resources. The analysis results indicate that collaboration strategies based on effort-based incentives work better than those using contribution-based incentives. The results also show that the use of effort-based incentives does not jeopardize the availability of local resources for the local needs.

Collaborative planning information delivery is an important issue in collaborative systems, but has not been well addressed in the research literature. Lino et al. proposed to integrate ontologies and reasoning mechanisms for multi-modality visualisation in collaborative planning environments. Ontologies and their integration support the expressiveness of several aspects related to real world applications in environments of mixed initiative planning from a visualisation perspective, while the reasoning mechanism allows a tailored delivery and visualisation of planning information. Since the proposed framework is based on the W3C standards, it facilitates communication and interoperability with other services and systems, and also supports extensions for its use on Semantic Web applications.

Service discovery and integration is an important research area with efforts invested to explore the potential advantages of collaborative computing. Zheng et al. proposed an Intents-based approach for service discovery and integration. Particularly, this paper proposed to use information retrieval techniques and applied them to intent resolving. An empirical approach was used through extensive experiments and analyses on a real dataset to obtain guiding principles based on which an adaptive intent resolving scheme was designed. 
López-Gil et al. presented a generic ontology called EmotionsOnto for describing emotions, which allows applications to identify and express them in applications. The ontology is proposed as a way to develop an easily computerizable and flexible formal model. Since it is based on the Web Ontology Language (OWL) standard, it is also easily shareable and extensible. The ontology has been validated through an application based on an emotions-aware Tangible User Interface (TUI).

Evolved from social networks and ad-hoc networks, opportunistic networks have been evolving quickly in recent years. The concept of "community", as one of the most inherent attributes of opportunistic networks, has been proved to be very helpful in simulating mobility traces of human society and selecting suitable message forwarders. Fu et al. developed an interest-driven community-based mobility model by considering location preference and time variance in human behaviour patterns. Based on this enhanced mobility model, a two-layer routing algorithm is proposed by jointly considering utilities generated by users' active degree and social relationships. Simulation results shown that the proposed routing scheme is able to improve delivery ratio while keeping the routing overhead and transmission delay within a reasonable range with respect to well-known routing schemes for opportunistic networks.

Yang et al. proposed a model for measuring achieved network capacity in multi-radio wireless mesh network (MR-WMNs) and proved that finding an optimal overlapping channel assignment in a given MR-WMN with an odd number of channels is equivalent to finding an optimal assignment by only using its orthogonal channels. This facilitates the use of fewer channels to solve complicated channel assignment problems. The effectiveness of the proposed approach has been validated through extensive simulations.

\section{Review Committee}

We would like to express our gratitude to the review committee members involved in this special issue for their valuable work on reviewing all the contributions.

Rachid Anane, Coventry University, UK

Pedro Antunes, Escola Superior de Tecnologia de Setúbal, Portugal

Nelson Baloian, University of Chile, Chile

Marcos Borges, Federal University of Rio de Janeiro, Brazil

Ruth Cobos, University of Madrid, Spain

Giancarlo Fortino, University of Calabria, Italy

Ning Gu, Fudan University, China

Tomoo Inoue, University of Tsukuba, Japan

Rahat Iqbal, Coventry University, UK

Yinsheng Li, Fudan University, China

Min Liu, Tongji University, China

Ta-Ping Lu. National Taipei University of Technology

Vincent Ng, Hong Kong Polytechnic University, China

Sergio F. Ochoa, University of Chile, Chile 
Jonice Oliveira, Federal University of Rio de Janeiro, Brazil

Hwa Gyoo Park, SCH University, Korea

Milton Pires Ramos, TECPAR, Brazil, China

Flavia Santoro, Rio de Janeiro State Federal University, Brazil

Jun Shen, University of Wollongong, Australia

Yuqing Sun, Shandong University, China

Cesar Tacla, SULBBS, Brazil

WenAn Tan, Nanjing University of Aeronautics and Astronautics, China

Adriana Vivacqua, Federal University of Rio de Janeiro, Brazil

Jun Yan, University of Wollongong, Australia

Jianming Yong, University of Southern Queensland, Australia

Haibin Zhu, Nipissing University, Canada

Weiming Shen

Weidong Li

Jose Alberto Pino

Junzhou Luo

Guest Editors

November 2014 